\title{
Composition and function of rhizosphere microbiome of Panax notoginseng with discrepant yields
}

\author{
Mengzhi Li ${ }^{1,2}$, Zhongjian Chen ${ }^{3,4}$, Jun Qiann ${ }^{2,5}$, Fugang Wei ${ }^{4}$, Guozhuang Zhang ${ }^{2}$, Yong Wang ${ }^{3}$, Guangfei Wei ${ }^{2}$, \\ Zhigang $\mathrm{Hu}^{1}$, Linlin Dong ${ }^{2^{*}}$ and Shilin Chen ${ }^{1,2^{*}}$
}

\begin{abstract}
Background: Panax notoginseng is a highly valuable medicinal plant. Reduced P. notoginseng yield is a common and serious problem that arises in a continuous cropping system. Variation in the composition and function of soil microbial community is considered the primary cause of yield reduction.

Methods: This study used shotgun metagenomic sequencing approaches to describe the taxonomic and functional features of $P$. notoginseng rhizosphere microbiome and screen microbial taxa and functional traits related to yields.

Results: At the family and genus level, a total of 43 families and 45 genera (relative abundance $>0.1 \%$ ) were obtained, and the correlation with the yield of P. notoginseng was further analyzed. Nitrosomonadaceae, Xanthomonadaceae, Mycobacterium and Arthrobacter that were enriched in soils with higher yields were positively correlated with P. notoginseng yields, thereby suggesting that they might increase yields. Negative correlation coefficients indicated that Xanthobacteraceae, Caulobacteraceae, Oxalobacteraceae, Chitinophagaceae, Sphingomonas, Hyphomicrobium, Variovorax and Phenylobacterium might be detrimental to $P$. notoginseng growth. A total of 85 functional traits were significantly $(P<0.05)$ correlated with $P$. notoginseng yields. Functional traits, likely steroid biosynthesis and MAPK signaling pathway were positively correlated with $P$. notoginseng yields. In contrast, functional traits, such as bacterial secretion system, ABC transporters, metabolism of xenobiotics by cytochrome P450 and drug metabolismcytochrome P450, were negatively associated with yields.
\end{abstract}

Conclusions: This study describes an overview of the rhizosphere microbiome of P. notoginseng with discrepant yields and identifies the taxa and functional traits related to yields. Our results provide valuable information to guide the isolation and culture of potentially beneficial microorganisms and to utilize the power of the microbiome to increase plant yields in a continuous cropping system.

Keywords: Rhizosphere microbiome, Panax notoginseng, Metagenome, Yields, Continuous cropping, Functional traits

*Correspondence: Ildong@icmm.ac.cn; slchen@icmm.ac.cn

${ }^{2}$ Key Laboratory of Beijing for Identification and Safety Evaluation

of Chinese Medicine, China Institute of Chinese Materia Medica, Academy of Chinese Medical Sciences, Beijing 100700, China

Full list of author information is available at the end of the article

\section{Background}

Sanchi ginseng [Panax notoginseng (Burk.) F. H. Chen] belongs to the Araliaceae family and is well known for its therapeutic effects [1-3], such as antihypertensive, antithrombotic, anti-atherosclerotic, anti-tumour, anti-oxidant and hepatoprotective activities [4-7]. P. notoginseng is used to treat cardiovascular diseases, inflammation, various body pains, trauma and internal and external bleeding due to injury [8]. At present, 
P. notoginseng roots are used as raw materials in more than 400 Chinese medicinal products of 1300 companies in China [9]. This species is in high demand annually in global market as a valuable traditional medicine. However, wild $P$. notoginseng resources have dwindled, and $P$. notoginseng is cultivated to satisfy market demands. $P$. notoginseng is a perennial medicinal plant cultivated in fixed plots for at least 3 years to obtain high quality roots $[10,11]$, and continuous cropping can decrease tuber quality and yield [12]. P. notoginseng has a narrow ecological range, and its cultivation primarily occurs in Wenshan, Yunnan Province [11, 13]. Approximately $8-10$ years of crop rotation is needed to replant $P$. notoginseng $[11,14]$. Nowadays, arable soils for $P$. notoginseng cultivation are becoming scarce [11]. The continuous cropping obstacle is a major constraint that hinders the sustainable development of $P$. notoginseng industry, thereby requiring urgent resolution.

Various factors have been considered to contribute to continuous cropping obstacle, including deterioration of soil physicochemical properties, soil-borne diseases, nutrient imbalance, changes in soil microbial communities and accumulation of autotoxic substances [15-18]. Soil microorganisms play an important role in soil function, especially in regulating plant growth, yield and quality $[19,20]$. The diversity and composition of soil microbial communities are critical to maintain soil health $[21,22]$. Many previous findings have demonstrated that continuous cropping is commonly linked to the disruption of the soil microbial community and regarded as a cause of reduced yield in Pisum sativum L., Rehmannia glutinosa, Arachis hypogaea L. and Malus pumila [16, 23-25]. Changes in soil microbial diversity and composition from continuous cropping system negatively affect soil productivity and crop yield $[25,26]$. Continuous monoculture of peanut increased pathogenic fungal and simplified beneficial fungal community, thereby contributing to the decline in peanut growth and yield [23]. The balance of soil microbial communities was disrupted in the continuous cropping practices of $P$. notoginseng. Moreover, the bacterial number and diversity in rhizosphere soil of $P$. notoginseng decreased with the increasing duration of continuous cropping [9]. Previous studies have focused on the changes of rhizosphere microorganisms in $P$. notoginseng in a continuous cropping system. However, information about soil microbial taxa related to $P$. notoginseng yield is rare. Identification of beneficial microbial communities that are relevant to plant yield contributes to the exploitation of the functional microbe to promote soil microecological environment in a continuous cropping system.

Amplicon sequencing approach is a classical method used to analyse microbial diversity and composition [27], thereby revealing the variations of microbial community in soils that underwent continuous cropping [14, 24-26]. Metagenomic shotgun sequencing can also determine the identity and relative abundance of microbes that are undetectable using amplicon sequencing approaches due to primer bias [27, 28]. Metagenomic analysis can provide taxonomic, genomic and functional information of the entire community of microorganisms at a given site $[27,29]$. However, the relatively high costs of shotgun metagenomics and more demanding bioinformatic requirements have precluded its use for microbiome analysis on a wide scale [30,31]. Many functional traits primarily involved in plant-microbe and microbemicrobe interactions are closely related to plant health and growth, and have been detected using metagenomic sequencing analysis [32]. Metagenomic sequencing analysis effectively reveals the microbial function of soil microbiome to reflect the ecological process in agricultural practice. Currently, the profiles of rhizosphere microbiome in the continuous cropping system using metagenomic sequencing analysis is rarely reported. A comprehensive understanding of rhizosphere microbial composition and function of $P$. notoginseng with discrepant yields will have significant agricultural implications, including maximizing the use of beneficial microbes and microbial derivatives to increase the yield of medicinal plants.

In this study, the structure and function of rhizosphere microbiome in the soils of $P$. notoginseng plants with different yields were investigated using metagenomic sequencing. We specifically aimed to do the following: (i) clarify the diversity, composition and function of soil microbiome in P. notoginseng plants; and (ii) elaborate the relationships among the microbial community and functional traits and $P$. notoginseng yield. Our study presented a comprehensive taxonomic and functional analysis of $P$. notoginseng rhizosphere microbiome by performing metagenomic sequencing, thereby establishing a foundation for the harnessing of the microbiome to improve $P$. notoginseng yield in a continuous cropping system.

\section{Materials and methods}

\section{Experimental design and sample collection}

Roots and rhizosphere soils were collected at $P$. notoginseng harvest stages (3-year-old root growth stages) to explore the relationship between rhizosphere microbiome and yields. P. notoginseng roots and rhizosphere soils were collected from Pingba $\left(23^{\circ} 14^{\prime} 29.5^{\prime \prime} \mathrm{N}, 104^{\circ} 5^{\prime} 3.0^{\prime \prime} \mathrm{E}\right.$, $1767 \mathrm{~m}$ a.s.l.), Yanshan $\left(23^{\circ} 34^{\prime} 56.22^{\prime \prime} \mathrm{N}, 104^{\circ} 19^{\prime} 49.05^{\prime \prime} \mathrm{E}\right.$, $1554 \mathrm{~m}$ a.s.l.) and Qiubei $\left(23^{\circ} 49^{\prime} 46.99^{\prime \prime} \mathrm{N}, 104^{\circ} 06^{\prime} 12.99^{\prime \prime} \mathrm{E}\right.$, $1631 \mathrm{~m}$ a.s.l.), Yunnan Province, China, where are the main production regions of $P$. notoginseng. Three experimental sites in the region of Pingba, namely, Pingba A, 
Pingba $\mathrm{B}$ and Pingba $\mathrm{C}$, were used. P. notoginseng was cultivated in strict accordance with the standard operating procedures established by the Good Agriculture Practices $[33,34]$. The 1-year-old $P$. notoginseng seedlings were transplanted in a plantation and cultivated for 2 years before harvest. The experiment was conducted as follows: block design with three replicates in each site; the area of each replicated plot was $1.4 \mathrm{~m} \times 10 \mathrm{~m}$ under the same management [2]. Seedlings were removed from $2 \mathrm{~m}^{2}$ of each plot, and fresh root weights were analysed to evaluate the $P$. notoginseng yield [35]. The yield was calculated for each plot as the number of weight $(\mathrm{kg})$ divided by the area of each plot $\left(\mathrm{m}^{2}\right)$. Data represent the mean of triplicates. In brief, $P$. notoginseng plants were gently removed from the soils, and rhizospheres were collected by gently shaking the roots to dislodge small adhering soil clumps [36]. Rhizosphere samples were randomly collected from healthy $P$. notoginseng roots (10 plants) in each plot and mixed to form a composite sample. Three replicates were utilised in one site. In total, 15 soil samples were collected, passed through a sieve $(2.0 \mathrm{~mm})$ and stored at $-80{ }^{\circ} \mathrm{C}$ for DNA extraction.

\section{DNA extraction and metagenome sequencing}

Total genomic DNA was extracted from $0.5 \mathrm{~g}$ of soil samples using the MoBio Powersoil Kit (MoBio Laboratories Inc., Carlsbad, CA, USA) according to the manufacturer's instructions. The DNA quantity and quality of each sample was determined by using a NanoDrop 2000 spectrophotometer (Thermo Scientific, USA) and electrophoresis (1.0\% agarose gel, including a $1 \mathrm{~kb}$ plus ladder). The DNA samples were stored at $-80^{\circ} \mathrm{C}$ until use. Metagenomic library preparation and sequencing were performed following the manufacturer's protocol at Biozeron, Shanghai, China. Fifteen rhizosphere soil DNA were selected for metagenomic sequencing to evaluate the microbial community structure and function. Metagenomic libraries were constructed using a TruSeq ${ }^{\mathrm{TM}}$ DNA Sample Prep Kit (Illumina, USA) according to the manufacturer's protocol. The metagenomic DNA was sonicated to the $450 \mathrm{bp}$ size range using a Covaris M220. The metagenomic libraries were sequenced on a HiSeq 2500 sequencer (Illumina, USA), and 150-bp paired-end reads were generated.

\section{Metagenomic analysis}

The raw reads from metagenome sequencing were used to generate clean reads by removing adaptor sequences and trimming and removing low quality reads (reads with an $\mathrm{N}$ base threshold of 10 and a minimum quality threshold of 20). The clean reads were further trimmed using Sickle software (https://github.com/najoshi/sickl e), and trimmed reads that were shorter than $75 \mathrm{bp}$ were discarded. The trimmed reads were mapped to $P$. notoginseng genome using Bowtie2 software [37] to identify and remove the $P$. notoginseng host-originated reads. The optimised sequence reads were assembled de novo by SOAPdenovo (http://soap.genomics.org.cn/, Version 1.06) based on a de Bruijn graph for obtaining contigs. The metagenes were predicted using MetaProdigal (http://prodigal.ornl.gov/) [38]. The non-redundant gene categories (unigenes) were generated using CD-HIT with an identity and average cut-off of $95 \%$ and $90 \%$, respectively [39]. The protein sequences were aligned against the NCBI microbial NR database using DIAMOND software [40] with an $E$ value cut-off of 1e-5 to generate the taxonomic information of the unigenes. Then, the taxonomic annotations of the unigenes were assigned using the MEGAN LCA algorithm [41]. The functional annotation was assigned to the unigenes by blasting against the KEGG database using BLASTP (BLAST v. 2.2.28+, http://blast.ncbi.nlm.nih.gov/Blast.cgi) $(e$ value $\leq 1 e-5)$.

\section{Statistical analysis}

Statistically significant differences in P. notoginseng yields, alpha diversity, phylum, family, genus and functional trait were examined using ANOVA test in SPSS 21.0 software (SPSS Institute, Inc., 2010). Significant differences were considered as $P<0.05$. Alpha diversity (Chao1 and Shannon index) was calculated using QIIME (http://qiime .org/index.html). The taxonomic and functional dissimilarity analyses in $P$. notoginseng rhizosphere soils with different yields were performed using $R$ package "vegan" with the Bray-Curtis metric. Principal coordinates analysis ( $\mathrm{PCoA}$ ) was performed using the $R$ package "stats" with the Bray-Curtis metric. Pearson's correlation analysis was performed to correlate the $P$. notoginseng yields with the abundance of the microbial taxa and functional traits using SPSS 21.0 software.

\section{Results}

\section{P. notoginseng yields in different sites}

$P$. notoginseng yields were significantly diverse in different sites in the range of $0.10-1.35 \mathrm{~kg} \mathrm{~m}^{-2}$ (Fig. 1). P. notoginseng yields were $0.10,0.25,0.68,0.76$ and $1.35 \mathrm{~kg} \mathrm{~m}^{-2}$ in Pingba A (PBA), Pingba B (PBB), Pingba C (PBC), Yanshan (YS) and Qiubei (QB), respectively. The yield was markedly higher in $\mathrm{QB}$ than those of $\mathrm{PBA}, \mathrm{PBB}, \mathrm{PBC}$ and YS $(P<0.001)$.

\section{Diversity of $P$. notoginseng rhizosphere microbiome}

To obtain more information about the composition and function of rhizosphere microbial community of $P$. notoginseng with different yields, rhizosphere DNA was selected for shotgun metagenomic sequencing via a HiSeq 2500 sequencer, after which a total of 


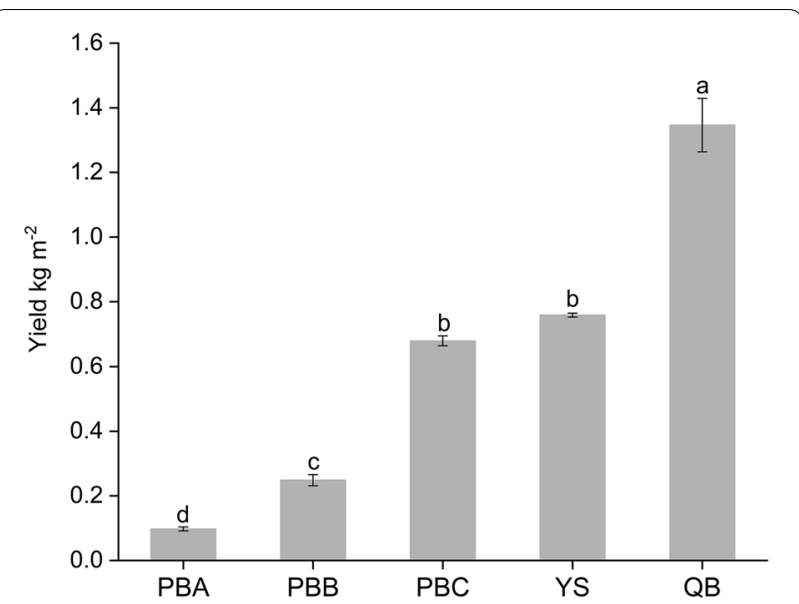

Fig. 1 . notoginseng yields. Different letters represent the significant differences between the sample sites at 0.05 significance level. Data represent the mean $\pm \mathrm{SE}(n=3)$

$1,104,569,780$ paired-end clean reads were obtained with approximately 62.9-86.6 million clean reads were obtained per sample (Additional file 1: Table S1). A total of 6,651,849 contigs were generated, with the longest contig at 611,672 bp and N50 at 1083 bp (Additional file 1: Table S2). After removing redundant sequences (identity $>95 \%$ and coverage $>90 \%$ ), 6,228,225 unigenes with an average length of $546.43 \mathrm{bp}$ were generated. The alpha diversity (Chao1 and Shannon index) of the rhizosphere microbiome showed the difference in the sample sites (Table 1). Shannon index at phylum, class, order and family levels were higher in the soils of YS and QB than that in the soils of PBA, PBB and PBC.

\section{Composition of $P$. notoginseng rhizosphere microbiome}

PCoA was performed based on metagenomic sequencing using the Bray-Curtis metric to visualise the difference in microbial communities among soil samples, thereby revealing significant difference in the microbial community in the rhizosphere soils using adonis test analysis $\left(\mathrm{R}^{2}=0.42, P=0.001\right)$ (Fig. 2a). The first principal component axis (18.7\% contributions) demonstrated that the microbial communities in the soils of YS and QB differed from those of the soils of PBA, PBB and PBC; the second principal component (13.61\% contributions) suggested that the microbial communities in the soils of YS significantly differed from those of other sites.

Bacteria is the predominant domain $(97.32 \% \pm 0.90 \%$, mean relative abundance $\pm S D, n=15$ ), with small proportions of Eukaryota, Archaea and Viruses detected based on the annotated unigenes. Proteobacteria, Actinobacteria, Acidobacteria, Chloroflexi, Bacteroidetes, Gemmatimonadetes and Verrucomicrobia were present at high relative abundances $(>1.0 \%)$ in the rhizosphere microbiome of sample sites at the phylum level (Fig. 2b). The relative abundance of Proteobacteria was $67.29 \%, 67.34 \%, 66.42 \%, 48.05 \%$ and $54.57 \%$, respectively, in rhizosphere soils of PBA, PBB, PBC, YS and QB. The relative abundance of Bacteroidetes was $3.73 \%, 5.68 \%$, $2.95 \%, 2.30 \%$ and $1.95 \%$, respectively, in rhizosphere soils of PBA, PBB, PBC, YS and $\mathrm{QB}$. The relative abundance of Proteobacteria and Bacteroidetes were significantly higher in rhizosphere soils of $\mathrm{PBA}, \mathrm{PBB}$ and $\mathrm{PBC}$ than YS and QB $(P<0.01)$. The relative abundance of Actinobactera was significantly higher in rhizosphere soils of YS (17.62\%) and QB (16.91\%) than PBA (8.43\%), PBB (7.93\%) and $\mathrm{PBC}(9.36 \%)(P<0.001)$.

Table 1 The alpha diversity (Chao1 and Shannon index) of the microbial communities revealed by metagenome data

\begin{tabular}{|c|c|c|c|c|c|}
\hline Sample sites & Phylum & Class & Order & Family & Genus \\
\hline \multicolumn{6}{|l|}{ Chao1 } \\
\hline PBA & $174.01 \pm 3.45^{\mathrm{ab}}$ & $291.92 \pm 3.76^{\mathrm{a}}$ & $495.19 \pm 2.66^{a}$ & $901.77 \pm 1.59^{\mathrm{a}}$ & $2654.37 \pm 9.43^{\mathrm{a}}$ \\
\hline PBB & $159.75 \pm 3.72^{b}$ & $283.89 \pm 9.67^{\mathrm{a}}$ & $498.07 \pm 12.24^{\mathrm{a}}$ & $883.88 \pm 15.11^{\mathrm{a}}$ & $2640.47 \pm 49.43^{\mathrm{a}}$ \\
\hline PBC & $166.37 \pm 4.47^{\mathrm{ab}}$ & $278.42 \pm 8.48^{\mathrm{a}}$ & $485.99 \pm 10.88^{\mathrm{a}}$ & $887.60 \pm 28.37^{a}$ & $2662.11 \pm 54.94^{a}$ \\
\hline YS & $172.20 \pm 6.72^{\mathrm{ab}}$ & $290.10 \pm 3.85^{\mathrm{a}}$ & $506.66 \pm 6.37^{\mathrm{a}}$ & $922.45 \pm 16.83^{\mathrm{a}}$ & $2680.45 \pm 18.87^{a}$ \\
\hline $\mathrm{QB}$ & $176.32 \pm 3.30^{\mathrm{a}}$ & $293.71 \pm 5.05^{\mathrm{a}}$ & $510.46 \pm 17.58^{\mathrm{a}}$ & $938.83 \pm 25.37^{a}$ & $2660.01 \pm 6.08^{\mathrm{a}}$ \\
\hline \multicolumn{6}{|l|}{ Shannon index } \\
\hline PBA & $1.99 \pm 0.02^{b}$ & $3.40 \pm 0.03^{b}$ & $4.85 \pm 0.03^{c}$ & $5.91 \pm 0.02^{b}$ & $7.27 \pm 0.03^{\mathrm{a}}$ \\
\hline PBB & $1.99 \pm 0.02^{b}$ & $3.34 \pm 0.07^{b}$ & $4.76 \pm 0.07^{c}$ & $5.86 \pm 0.05^{b}$ & $7.30 \pm 0.04^{\mathrm{a}}$ \\
\hline PBC & $2.01 \pm 0.12^{b}$ & $3.34 \pm 0.15^{b}$ & $4.77 \pm 0.10^{c}$ & $5.85 \pm 0.09^{b}$ & $7.24 \pm 0.06^{\mathrm{a}}$ \\
\hline YS & $2.63 \pm 0.03^{a}$ & $3.97 \pm 0.04^{\mathrm{a}}$ & $5.38 \pm 0.03^{\mathrm{a}}$ & $6.25 \pm 0.03^{\mathrm{a}}$ & $7.35 \pm 0.03^{\mathrm{a}}$ \\
\hline $\mathrm{QB}$ & $2.39 \pm 0.10^{a}$ & $3.70 \pm 0.08^{\mathrm{a}}$ & $5.14 \pm 0.09^{b}$ & $6.05 \pm 0.12^{\mathrm{ab}}$ & $7.18 \pm 0.18^{a}$ \\
\hline
\end{tabular}

PBA Pingba village A, PBB Pingba village B, PBC Pingba village $C, Y S$ Yanshan village, $Q B$ Qiubei village. The mean values of three replicates per site are show, followed by the standard error of the mean. Different letters represent significant difference among five sample sites at the level of 0.05 

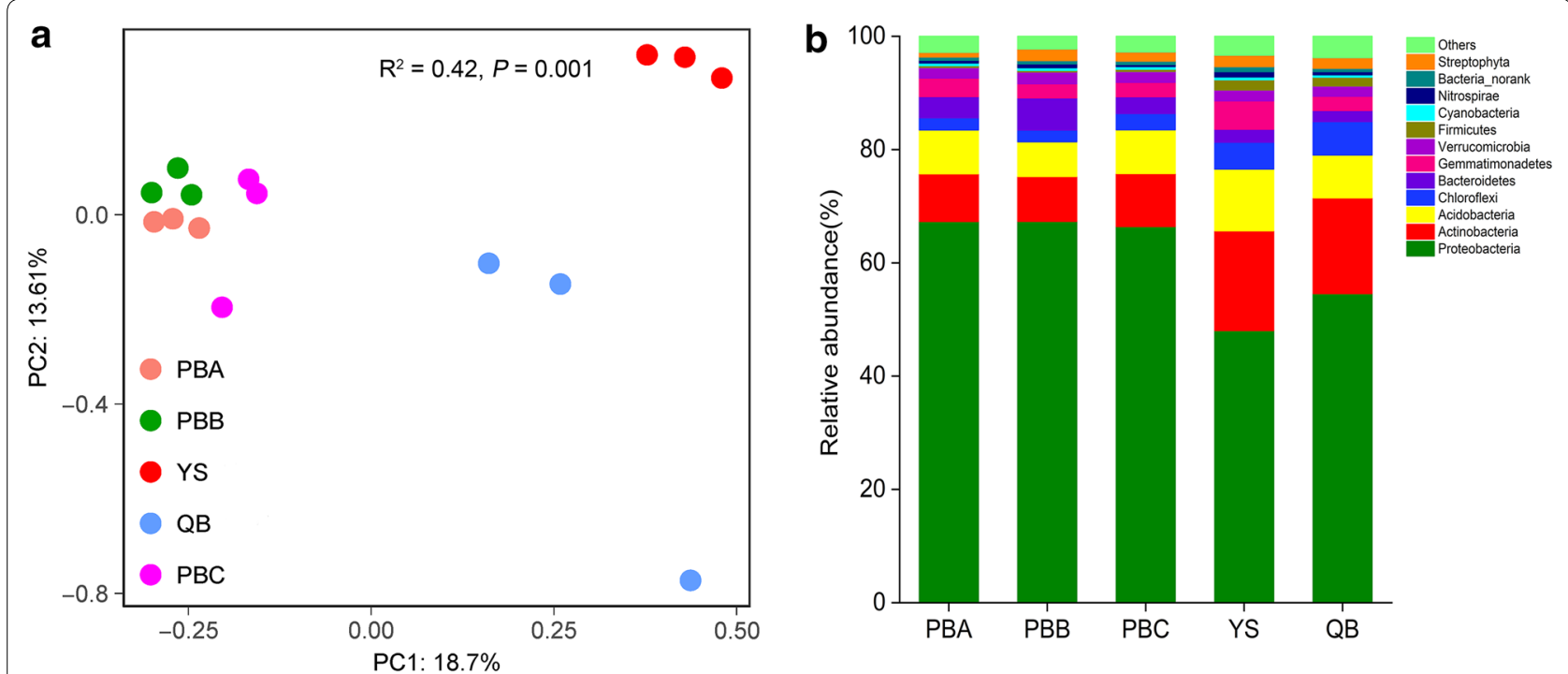

Fig. 2 Taxonomic composition of P. notoginseng rhizosphere microbiome. a PCoA based on the Bray-Curtis distance between different rhizosphere samples. b Relative abundance of different phyla in rhizosphere samples

\section{Correlations between taxonomic taxa and P. notoginseng yields}

Forty-three families (relative abundance>0.1\%) were obtained from rhizosphere soils of $P$. notoginseng (Fig. 3a). The relative abundance of Caulobacteraceae was $2.08 \%, 2.45 \%, 1.94 \%, 1.42 \%$ and $1.27 \%$, respectively, in rhizosphere soils of PBA, PBB, PBC, YS and QB. The relative abundance of Methylobacteriaceae was $0.21 \%, 0.18 \%$, $0.19 \%, 0.18 \%$ and $0.13 \%$, respectively, in rhizosphere soils of $\mathrm{PBA}, \mathrm{PBB}, \mathrm{PBC}, \mathrm{YS}$ and $\mathrm{QB}$. The relative abundance of Caulobacteraceae and Methylobacteriaceae were significantly lower in rhizosphere soils of QB (higher yields) than PBA, PBB, PBC and YS $(P<0.05)$. The relative abundance of Micrococcaceae was significantly higher in rhizosphere soils of QB (0.76\%) than PBA (0.49\%), PBB (0.40\%), PBC (0.21\%) and YS $(0.50 \%) \quad(P<0.05)$. Pearson's correlation analysis showed that the relative abundance of Comamonadaceae $(\mathrm{R}=-0.89)$, Opitutaceae $(R=-0.88)$, Rhodobacteraceae $(R=-0.96)$ and Sphingobacteriaceae $(R=-0.90)$ were negatively related to $P$. notoginseng yield $(P<0.05)$. Relative abundance of Ktedonobacteraceae $(\mathrm{R}=0.95)$, Streptosporangiaceae $(\mathrm{R}=0.91)$ and Thermomonosporaceae $(\mathrm{R}=0.90)$ were positively correlated to $P$. notoginseng yield $(P<0.05)$.

At the genus level, we obtained a total of 45 genera (relative abundance $>0.1 \%$ ) (Fig. $3 b$ ). The relative abundance of Leifsonia was significantly higher in rhizosphere soils of QB $(0.40 \%)$ than PBA (0.21\%), PBB (0.15\%), PBC $(0.17 \%)$ and YS $(0.20 \%)(P<0.01)$. The relative abundance of Pseudomonas was significantly higher in rhizosphere soils of PBA (lower yields, 1.55\%) than PBB (0.93\%), PBC
(0.60\%), YS $(0.90 \%)$ and QB $(1.48 \%)(P<0.05)$. Pearson's correlation analysis showed that the relative abundance of Actinomadura $(\mathrm{R}=0.89)$, Arthrobacter $(\mathrm{R}=0.41)$, Enhydrobacter $(\mathrm{R}=0.73)$, Leifsonia $(\mathrm{R}=0.79)$, Mycolicibacterium $(\mathrm{R}=0.83)$, Rhizophagus $(\mathrm{R}=0.74)$ were positively correlated with $P$. notoginseng yield. Whereas the relative abundance of Bosea $(\mathrm{R}=-0.85)$, Cupriavidus $(\mathrm{R}=-0.91)$, Mucilaginibacter $(\mathrm{R}=-0.89)$, Novosphingobium $(\mathrm{R}=-0.79)$, Phenylobacterium $(\mathrm{R}=-0.89)$, Opitutus $(\mathrm{R}=-0.85)$, Phenylobacterium $(\mathrm{R}=-0.89)$, Polaromonas $(\mathrm{R}=-0.82)$, Reyranella $(\mathrm{R}=-0.70)$, Rhizobium $(\mathrm{R}=-0.74)$, Sphingobium $(\mathrm{R}=-0.87)$, Sphingomonas $(\mathrm{R}=-0.83)$ and Variovorax $(\mathrm{R}=-0.85)$ were negatively correlated with $P$. notoginseng yield.

\section{The functional traits of the $P$. notoginseng rhizosphere microbiome}

In total, 1,330,812 genes were hit in the KEGG databases and were assigned to $4436 \mathrm{KEGG}$ orthology (KO) functional categories (Additional file 2). The KOs were mainly involved in 6 KEGG level 1 pathways and 43 KEGG level 2 pathways (Fig. $4 \mathrm{a}, \mathrm{b}$ ). The relative abundance of cellular processes, environmental information processing, genetic information processing, human diseases, metabolism and organismal systems pathways were $0.89 \%-1.24 \%, 2.56 \%-3.54 \%, 2.83 \%-3.72 \%, 1.60 \%-2.08 \%$, $39.62 \%-51.83 \%$ and $0.94 \%-1.09 \%$, respectively, in rhizosphere soils of five sample sites at the first KEGG level. The relative abundance of cellular processes, environmental information processing, genetic information processing and human diseases were significantly higher in 

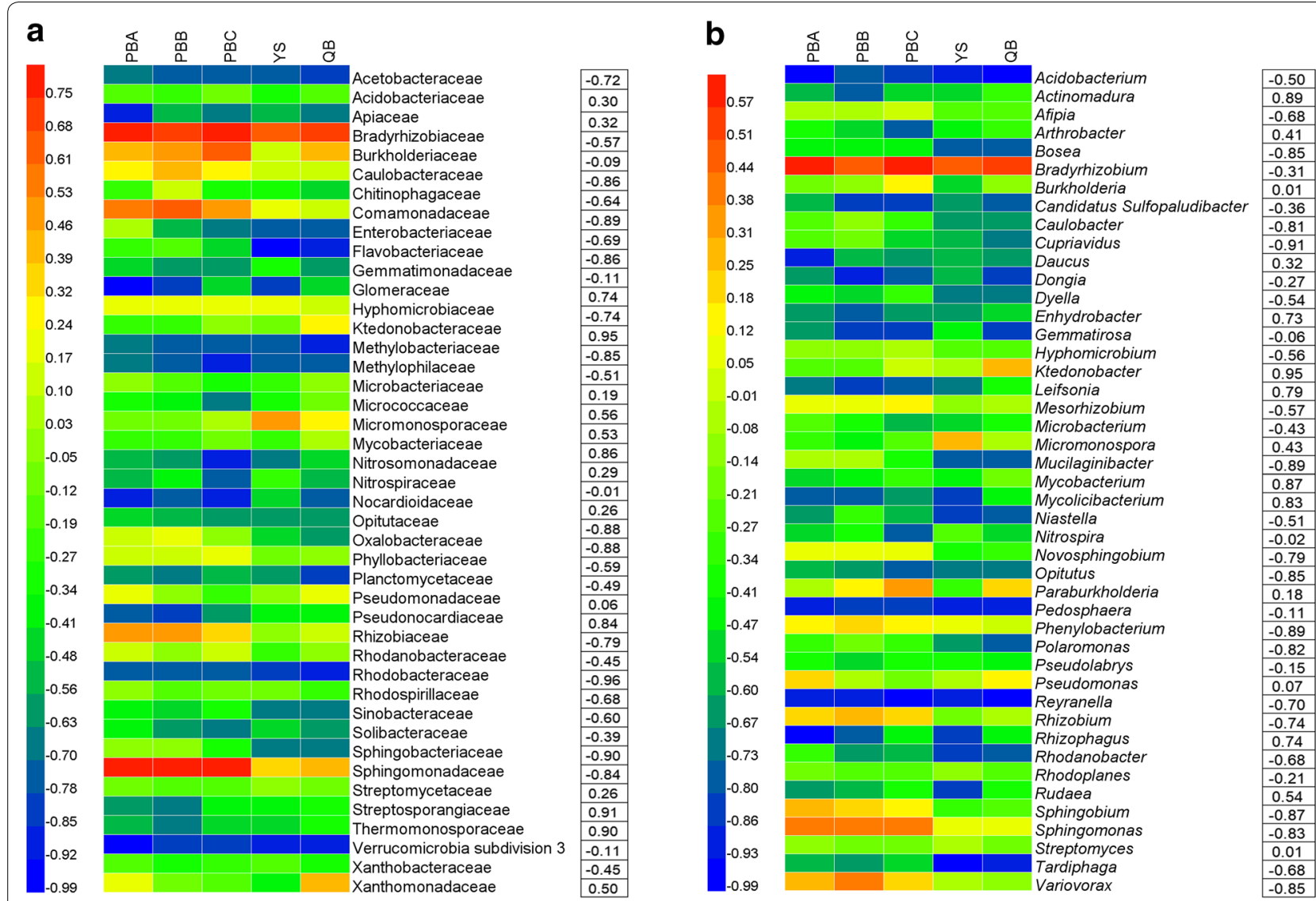

Fig. 3 Relative abundance $(>0.1 \%)$ of the dominant families $\mathbf{a}$ and genera $\mathbf{b}$, and their Pearson's correlation coefficients with $P$. notoginseng yields. Data are mean values of $n=3$

rhizosphere soils of PBA (lower yields) than PBB, PBC, YS and QB $(P<0.05)$. The relative abundance of biosynthesis of other secondary metabolites, drug resistance, environmental adaptation, glycan biosynthesis and metabolism, lipid metabolism, metabolism of cofactors and vitamins, membrane transport and signal transduction were $0.46 \%-0.61 \%, 0.28 \%-0.41 \%, 0.05 \%-0.07 \%$, $0.40 \%-0.55 \%, 1.24 \%-1.63 \%, 1.53 \%-2.10 \%, 1.38 \%-1.98 \%$ and $1.18 \%-1.56 \%$, respectively, in rhizosphere soils of five sample sites at the second KEGG level. The relative abundance of biosynthesis of other secondary metabolites, drug resistance, environmental adaptation, glycan biosynthesis and metabolism, metabolism of cofactors and vitamins, membrane transport and signal transduction were significantly higher in rhizosphere soils of PBA (lower yields) than $\mathrm{PBB}, \mathrm{PBC}, \mathrm{YS}$ and $\mathrm{QB}(P<0.05)$. The relative abundance of lipid metabolism was significantly lower in rhizosphere soils of $\mathrm{QB}$ (higher yields) than PBA, PBB, PBC and YS $(P<0.05)$.

PCoA was performed based on KO functional categories using the Bray-Curtis metric, and an adonis test $\left(\mathrm{R}^{2}=0.62, P=0.001\right)$ showed significant difference in sample sites (Fig. 4c). KO functional categories with a relative abundance exceeding 0.15\% are described in Fig. $4 \mathrm{~d}$ to clarify which $\mathrm{KO}$ functional categories were dominant among rhizosphere microbiome of $P$. notoginseng and which metabolism pathways were the main components in sample sites. Ninety-three KO functional categories were obtained with differences among the sample sites. The K01999 (mean 0.61\%), K00626 (mean 0.50\%), K00799 (mean 0.43\%), K00059 (mean 0.42\%) and K03701 (mean $0.40 \%$ ) were the top five categories that were enriched in rhizosphere soils of PBA, PBB, PBC, YS and QB. Notably, the relative abundance of K01999 was the highest among all the KO functional categories, and K00626 was the second highest. K01999, a branched-chain amino acid transport system substrate-binding protein, is a member of membrane transport pathway. K00626 (atoB, acetylCoA C-acetyltransferase) is involved in carbon metabolism, pyruvate metabolism, carbon fixation pathways in prokaryotes, two-component system, fatty acid metabolism and biosynthesis of antibiotics pathways. K00799 (gst, glutathione S-transferase) is involved in glutathione metabolism, metabolism of xenobiotics by cytochrome 

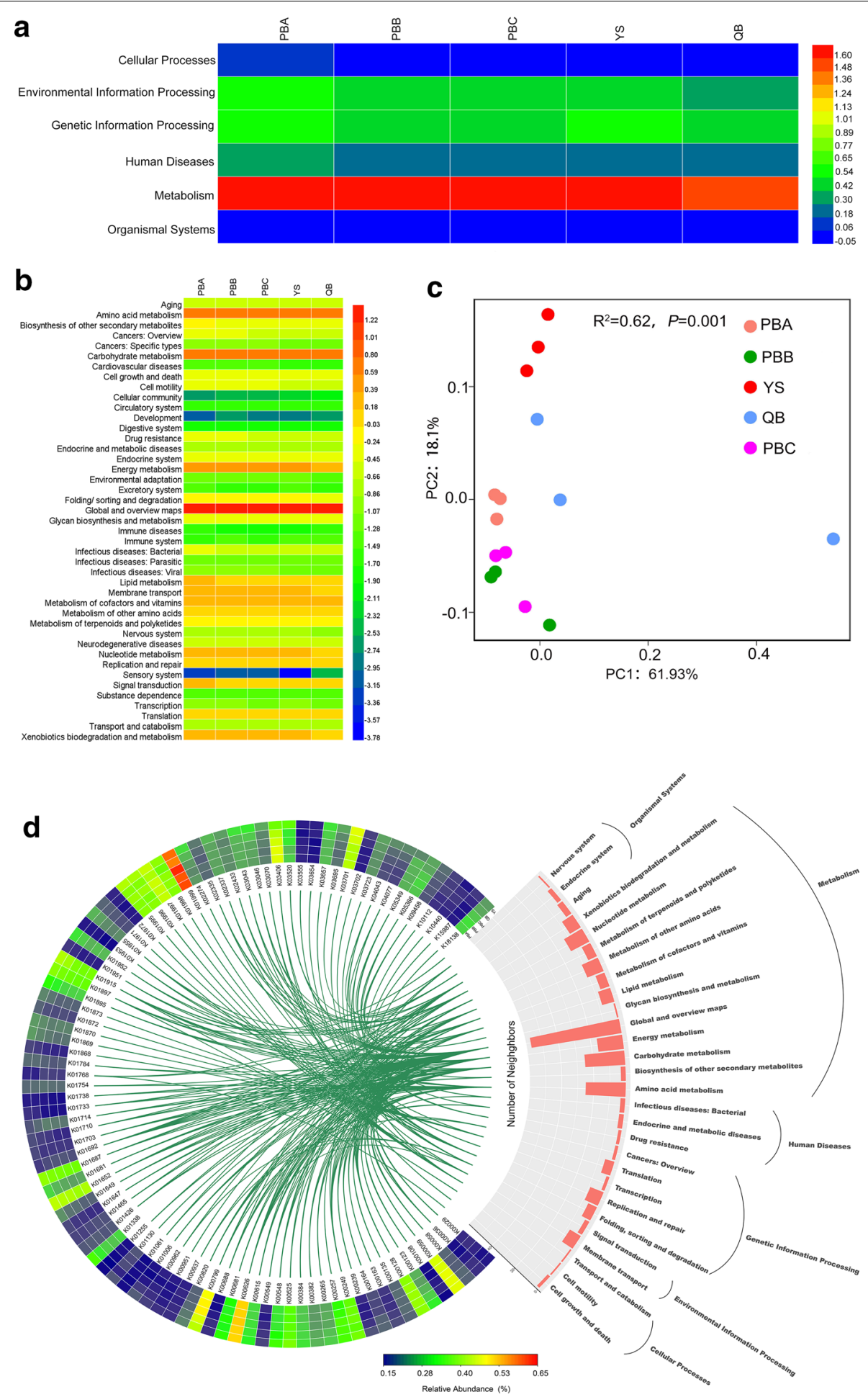

Fig. 4 Characterisation of the functional traits of $P$. notoginseng rhizosphere microbiome. a Functional traits in KEGG level 1 pathway. b Functional traits in KEGG level 2 pathway. $\mathbf{c}$ The principal coordinates analysis (PCOA) of KO functional categories. $\mathbf{d}$ The relative abindance $(\geq 0.15 \%)$ of KO functional categories. Data are mean values of $n=3$ 
P450 and drug metabolism-cytochrome P450 pathways. K00059 (fabG, 3-oxoacyl-[acyl-carrier protein] reductase) is involved in metabolic, fatty acid metabolism, fatty acid biosynthesis, biosynthesis of unsaturated fatty acids and biotin metabolism pathways. K03701 (uvrA, excinuclease $A B C$ subunit $A$ ) is mainly involved in the nucleotide excision repair pathway.

\section{Correlations between functional traits and $P$. notoginseng yields}

A total of 389 functional classifications were obtained, and the relative abundance showed differences among the sample sites at the third KEGG level (Additional file 3 and Fig. 5). The relative abundance of functional traits, likely steroid hormone biosynthesis (ko00140), lysine biosynthesis (ko00300), ABC transporters (ko02010), two-component system (ko02020) and plant-pathogen interaction (ko04626) were significantly lower in rhizosphere soils of QB (higher yields) than PBA, PBB, PBC and YS $(P<0.05)$. Eighty-five functional traits were significantly correlated $(P<0.05)$ with $P$. notoginseng yields using Pearson's correlation analysis, among 12 and 73 functional traits were positively and negatively correlated with yields, respectively. A total of 304 functional traits were correlated with $P$. notoginseng yields, among which 140 and 164 functional traits were positively and negatively correlated with yields, respectively.

The KOs involved in known plant-microbe and microbe-microbe interactions, such as bacterial secretion system (ko03070), flagellar assembly (ko02040), bacterial chemotaxis (ko02030) and two-component system (ko02020), were negatively correlated with $P$. notoginseng yields (Additional file 3 and Fig. 5). Pearson's correlation analysis showed that the abundances of plant-pathogen interaction (ko04626), ABC transporters (ko02010), metabolism of xenobiotics by cytochrome P450 (ko00980), drug metabolism-cytochrome P450 (ko00982), dioxin degradation (ko00621), chloroalkane and chloroalkene degradation (ko00625) and degradation of aromatic compounds (ko01220) were negatively correlated with $P$. notoginseng yields. Meanwhile the abundances of MAPK signaling pathway (ko04010) and steroid biosynthesis (ko00100) were positively correlated with $P$. notoginseng yields.

\section{Discussion}

This study analysed the taxonomic and functional features of $P$. notoginseng rhizosphere microbiome with discrepant yields to determine microbial taxa and functional traits related to yields. Microbial composition exhibited dissimilarity in rhizosphere soils of $P$. notoginseng with discrepant yields, and the findings were consistent with those for other crops [24]. Actinobacteria was more abundant in disease-suppressive soils than in disease-conducive soils in continuous cropping system [42]. And we found that Actinobacteria was enriched in rhizosphere soils of $P$. notoginseng with high yields. Xanthobacteraceae, Caulobacteraceae, Oxalobacteraceae, Phyllobacteriaceae and Chitinophagaceae negatively correlated with Setaria italica yields [43]. Dongia, Opitutus, Sphingomonas, Rhizobium, Hyphomicrobium and Phenylobacterium were negatively correlated with apple rootstocks growth [44]. The above taxa were also negatively correlated with $P$. notoginseng yields in our study. Variovorax was positively correlated with the Solanum tuberosum L. common scab severity level and was supposedly responsible for inducing common scab by stimulating thaxtomin production [45]. Moreover, the high abundances of Variovorax were shown in soils with low P. notoginseng yields in our study. Overall, Variovorax might negatively influence the yields of $P$. notoginseng. Certain microbial communities in the rhizosphere soils could negatively regulate the growth and yields of plants.

Plant growth-promoting rhizobacteria (PGPR) play an important role in enhancing plant health, promoting plant growth and increasing crop yields [46, 47]. Some PGPR, such as Nitrosomonadaceae, Xanthomonadaceae, Arthrobacter and Mycobacterium, presented positive correlations with $P$. notoginseng yields in our study. Nitrosomonadaceae is used for bioremediation of toxic chemicals in the soil [48]. The addition of Nitrosomonadaceae could reduce nitrogen loss and the time required to stabilise the nitrogen profile $[48,49]$. Xanthomonadaceae was positively correlated with foxtail millet yields [43]. Mycobacterium, as PGPR genera, had positive effect on plant growth, nutrient uptake and increased root dry weight of maize $[46,47,50,51]$. Arthrobacter acts as antagonistic bacteria against Sclerotinia sclerotiorum [52], which can cause Helianthus annuus L. sclerotinia rot [53]. The abundance of Arthrobacter had significant negative correlation with tobacco bacterial wilt disease [54]. These results indicated that the enrichment of Mycobacterium and Arthrobacter in soils with higher yields might contribute to the increase of $P$. notoginseng yields. Therefore, regulation of rhizosphere microbiomes contributed to overcome continuous cropping obstacles and increase medicinal plant yields by improving soil environment.

A total of 83 microbial functional traits were significantly correlated $(P<0.05)$ with $P$. notoginseng yields. Moreover, these functional traits contained bacterial secretion system, $\mathrm{ABC}$ transporters, metabolism of xenobiotics by cytochrome P450 and drug metabolismcytochrome $\mathrm{P} 450$ which were negatively associated with $P$. notoginseng yields. $\mathrm{ABC}$ transporters and bacterial secretion system were enriched in soils of potato with high scab severity level [45], and those functional traits mediated the communication between microorganisms 


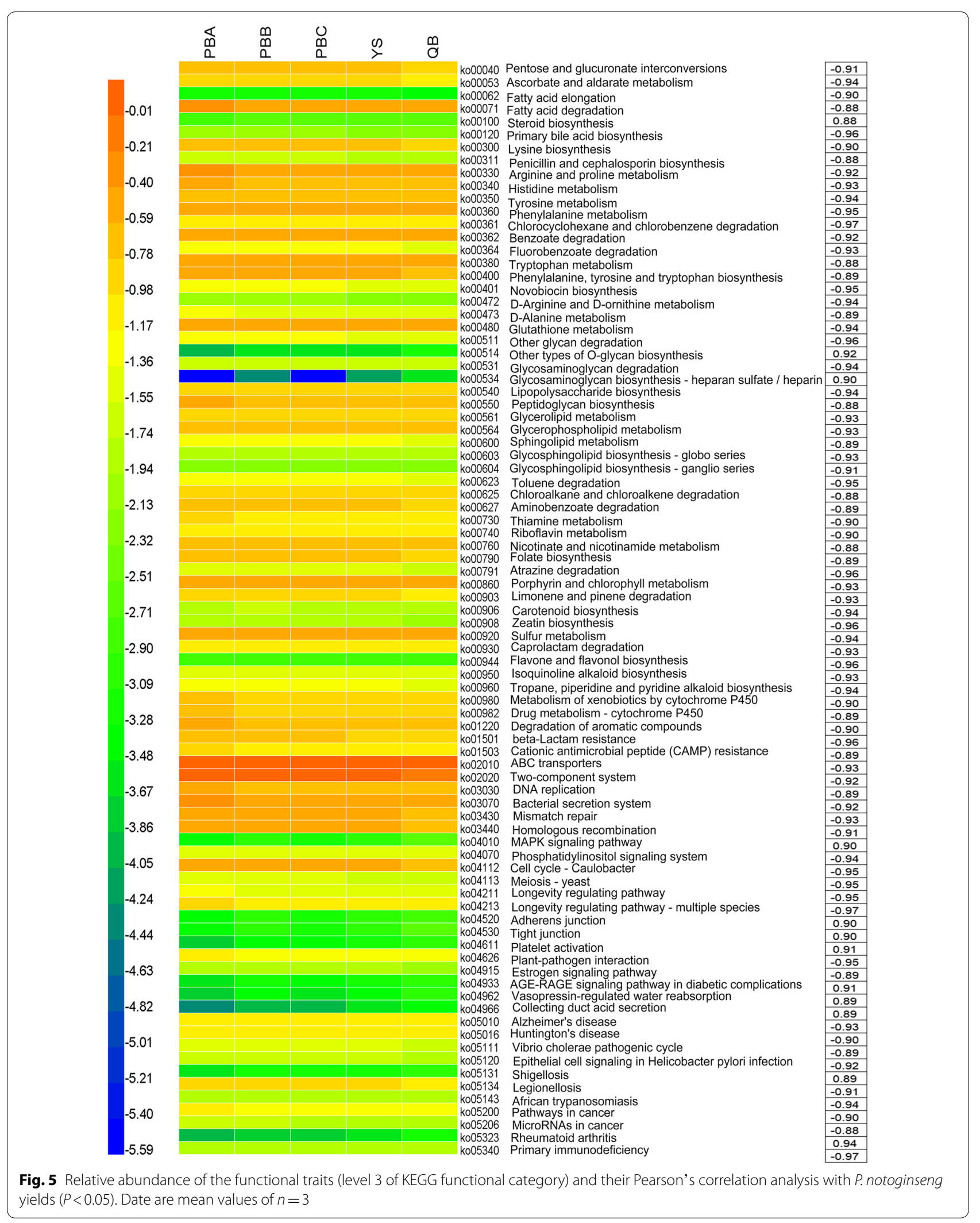


and environments or other organisms [55-57].Thaxtomins are highly phytotoxic cyclic dipeptides produced by plant-pathogenic members of the genus Streptomyces [58]. Thaxtomins have the basic structure L-4-nitrotryptophyl-L-phenylalanyl [59], and the biosynthetic pathway of Thaxtomin A (ThxA) involves nitric oxide synthase and cytochrome P450 [60-62]. In this study, the high abundance of xenobiotic metabolism by cytochrome P450, drug metabolism-cytochrome P450 and nitrogen metabolism in low yield soils might be conducive to ThxA biosynthesis. In summary, the occurrence of low $P$. notoginseng yields was accompanied by an increase in the abundance of pathogenicity-related functional traits in rhizosphere microbes. Functional traits involved in MAPK signaling pathway and steroid biosynthesis were positively correlated with $P$. notoginseng yields in the present study. In eukaryotic cells, MAPKs is involved in the transduction of a variety of extracellular signals and the regulation of different developmental processes, such as regulating fungal mating, invasive growth, cell wall integrity and ascospore formation [63, 64]. Steroids can act as signal molecules that mediate communication between microorganisms and hosts $[65,66]$. The functional traits involved in MAPK signaling pathway and steroid biosynthesis might benefit $P$. notoginseng yield.

\section{Conclusion}

In summary, the taxonomic and functional properties exhibited dissimilarity in rhizosphere microbiome of $P$. notoginseng with different yields in continuous cropping system based on the shotgun metagenomic sequencing. These microorganisms such as Arthrobacter and Mycobacterium, and functional traits involved in steroid biosynthesis and MAPK signaling pathway were positively correlated with yields. This work broadens the understanding of the relationship between the rhizosphere microbial composition and function with $P$. notoginseng yields and lays a foundation for the exploitation of microbes to improve soil microecological environment and increase medicinal plant yields in continuous cropping system.

\section{Supplementary information}

Supplementary information accompanies this paper at https://doi. org/10.1186/s13020-020-00364-4.

Additional file 1: Table S1. Summary of the metagenomic reads in five sample sites. Table S2. Statistical analysis of rhizosphere soils in P. notoginseng from five different sites.

Additional file 2. Abundance of the metagenomic microbial function profiling (KEGG orthology function category).

Additional file 3. Pearson correlation analysis $(P \geq 0.05)$ among functional traits regarding $P$. notoginseng yields.
Abbreviations

PCOA: Principal coordinates analysis; KEGG: Kyoto Encyclopedia of Genes and Genomes; KO: KEGG orthology; PGPR: Plant growth-promoting rhizobacteria.

\section{Acknowledgements}

We are particularly grateful to Jun Qian for his valuable comments on the data analysis.

\section{Authors' contributions}

SC and LD conceived and designed the research; ML, YW, FW, and ZH per-

formed the experiments; ML, ZC, JQ, GW, and GZ analyzed the data; ML and

LD wrote the manuscript. All authors read and approved the final manuscript.

\section{Funding}

This study was supported by grands from the Beijing Nova Program (No. Z181100006218020), the Fundamental Research Funds for the Central public welfare research institutes (No.ZZ13-AQ-049), the Major Science and Technology Programs in Yunnan Province (2018ZF011-3) and National Key R\&D Plan (No. 2017YFC1702500), National Science and Technology Major Project for "Significant New Drugs Development" (2019ZX09201005-002-001; 2019ZX09201005-006-001).

\section{Data availability statement}

The raw sequencing data are publicly available in the NCBI Sequence Read Archive (SRA) under the Bioproject Number PRJNA595820.

Ethics approval and consent to participate

Not applicable.

\section{Consent for publication}

Not applicable.

\section{Competing interests}

The authors declare no conflict of interest.

\section{Author details}

${ }^{1}$ College of Pharmacy, Hubei University of Chinese Medicine, Wuhan 430065, China. ${ }^{2}$ Key Laboratory of Beijing for Identification and Safety Evaluation of Chinese Medicine, China Institute of Chinese Materia Medica, Academy of Chinese Medical Sciences, Beijing 100700, China. ${ }^{3}$ Institute of Sanqi Research, Wenshan University, Wenshan 663000, China. ${ }^{4}$ Wenshan Miaoxiang Notoginseng Technology, Co., Ltd, Wenshan 663000, China. ${ }^{5}$ College of Pharmaceutical Science, Dali University, Dali 671000, China.

Received: 11 June 2020 Accepted: 3 August 2020

Published online: 10 August 2020

\section{References}

1. Yang M, Zhang XD, Xu YG, Mei XY, Jiang BB, Liao JJ, et al. Autotoxic ginsenosides in the rhizosphere contribute to the replant failure of Panax notoginseng. PLoS ONE. 2015;10(2):e0118555. https://doi.org/10.1371/ journal.pone.0118555.

2. Dong LL, Xu J, Feng GQ, Li XW, Chen SL. Soil bacterial and fungal community dynamics in relation to Panax notoginseng death rate in a continuous cropping system. Sci Rep. 2016;6:31802. https://doi.org/10.1038/srep3 1802.

3. Ma YN, Chen CJ, Li QQ, Xu FR, Cheng YX, Dong X. Monitoring antifungal agents of Artemisia annua against Fusarium oxysporum and Fusarium solani, associated with Panax notoginseng root-rot disease. Molecules. 2019;24(1):213. https://doi.org/10.3390/molecules24010213.

4. Chan P, Thomas GN, Tomlinson B. Protective effects of trilinolein extracted from Panax notoginseng against cardiovascular disease. Acta Pharmacol Sin. 2002;23(12):1157-62. https://doi.org/10.1021/ar010155r.

5. Ng TB. Pharmacological activity of sanchi ginseng (Panax notoginseng). J Pharm Pharmacol. 2006;58(8):1007-19. https://doi.org/10.1211/ jpp.58.8.0001.

6. Xiang H, Liu YX, Zhang BB, Huang JH, Li Y, Yang B, et al. The antidepressant effects and mechanism of action of total saponins from the caudexes and leaves of Panax notoginseng in animal models of 
depression. Phytomedicine. 2011;18(8-9):8. https://doi.org/10.1016/j. phymed.2010.11.014

7. Sun K, Fan JY, Han JY. Ameliorating effects of traditional Chinese medicine preparation, Chinese materia medica and active compounds on ischemia/reperfusion-induced cerebral microcirculatory disturbances and neuron damage. Acta Pharm Sin B. 2015;5(1):8-24. https://doi. org/10.1016/j.apsb.2014.11.002.

8. Sun HX, Qin F, Ye YP. Relationship between haemolytic and adjuvant activity and structure of protopanaxadiol-type saponins from the roots of Panax notoginseng. Vaccine. 2005;23(48-49):5533-42. https://doi. org/10.1016/j.vaccine.2005.07.036.

9. Tan Y, Cui YS, Li HY, Kuang AX, Li XR, Wei YL, et al. Diversity and composition of rhizospheric soil and root endogenous bacteria in Panax notoginseng during continuous cropping practices. J Basic Microbiol. 2017;57(4):337-44. https://doi.org/10.1002/jobm.201600464.

10. Guo HB, Cui XM, An N, Cai GP. Sanchi ginseng (Panax notoginseng (Burkill) F H Chen) in China: distribution, cultivation and variations. Genet Resour Crop Evol. 2010;57:453-60. https://doi.org/10.1007/s1072 2-010-9531-2.

11. Dong LL, Cheng RY, Xiao LN, Wei GF, Xu J, Wang Y, et al. Diversity and composition of bacterial endophytes among plant parts of Panax notoginseng. Chin Med. 2018;13:41. https://doi.org/10.1186/s1302 0-018-0198-5.

12. Dong LL, Yao H, Li QS, Song JY, Li Y, Luo HM, et al. Investigation and integrated molecular diagnosis of root-knot nematodes in Panax notoginseng root in the field. Eur J Plant Pathol. 2013;137:667-75. https://doi. org/10.1007/s10658--0277-5.

13. Yao L, Wang SH, Liang WX, Wang J, Gao WY. Screening and evaluation of adventitious root lines of Panax notoginseng by morphology, gene expression, and metabolite profiles. Appl Microbiol Biotechnol. 2019;103(11):4405-15. https://doi.org/10.1007/s00253-019-09778-z.

14. Zhang Y, Zheng YJ, Xia PG, Xun LL, Liang ZS. Impact of continuous Panax notoginseng plantation on soil microbial and biochemical properties. Sci Rep. 2019;9(1):13205. https://doi.org/10.1038/s41598-019-49625-9.

15. Berg G, Smalla K. Plant species and soil type cooperatively shape the structure and function of microbial communities in the rhizosphere. FEMS Microbiol Ecol. 2009;68(1):1-13. https://doi.org/10.111 1/j.1574-6941.2009.00654.x.

16. Nayyar A, Hamel C, Lafond G, Gossen BD, Hanson K, Germida J. Soil microbial quality associated with yield reduction in continuous-pea. Appl Soil Ecol. 2009;43:115-21. https://doi.org/10.1016/j.apsoil.2009.06.008.

17. Mondal MF, Asaduzzaman M, Kobayashi Y, Ban T, Asao T. Recovery from autotoxicity in strawberry by supplementation of amino acids. Sci Hortic. 2013;164:137-44. https://doi.org/10.1016/j.scienta.2013.09.019.

18. Ling N, Deng KY, Song Y, Wu YC, Zhao J, Raza W, et al. Variation of rhizosphere bacterial community in watermelon continuous mono-cropping soil by long-term application of a novel bioorganic fertilizer. Microbiol Res. 2014;169(7-8):570-8. https://doi.org/10.1016/j.micres.2013.10.004.

19. Berendsen RL, Pieterse CMJ, Bakker PAHM. The rhizosphere microbiome and plant health. Trends Plant Sci. 2012;17(8):478-86. https://doi. org/10.1016/j.tplants.2012.04.001

20. Andreote FD, Pereira ESMC. Microbial communities associated with plants: learning from nature to apply it in agriculture. Curr Opin Microbiol. 2017;37:29-34. https://doi.org/10.1016/j.mib.2017.03.011.

21. Acosta-Martínez V, Upchurch DR, Schubert AM, Porter D, Wheeler T. Early impacts of cotton and peanut cropping systems on selected soil chemical, physical, microbiological and biochemical properties. Biol Fertil Soils. 2004;40:44-54. https://doi.org/10.1007/s00374-004-0745-3.

22. Garbeva P, van Veen JA, van Elsas JD. Microbial diversity in soil: selection of microbial populations by plant and soil type and implication on disease suppressiveness. Annu Rev Phytopathol. 2004;42:243-70. https:// doi.org/10.1146/annurev.phyto.42.012604.135455.

23. Chen MN, Li X, Yang QL, Chi XY, Pan LJ, Chen N, et al. Soil eukaryotic microorganism succession as affected by continuous cropping of peanut- pathogenic and benefcial fungi were selected. PLOS ONE. 2012;7(7):e40659. https://doi.org/10.1371/journal.pone.0040659.

24. Wu LK, Wang JY, Huang WM, Wu HM, Chen J, Yang YQ, et al. Plant-microbe rhizosphere interactions mediated by Rehmannia glutinosa root exudates under consecutive monoculture. Sci Rep. 2016;6:19101. https://doi. org/10.1038/srep15871.
25. Jiang JH, Song Z, Yang XT, Mao ZQ, Nie XH, Guo H, et al. Microbial community analysis of apple rhizosphere around Bohai Gulf. Sci Rep. 2017;7(1):8918. https://doi.org/10.1038/s41598-017-08398-9.

26. Mazzola M, Manici LM. Apple replant disease: role of microbial ecology incause and control. Annu Rev Phytopathol. 2012;50:45-65. https://doi. org/10.1146/annurev-phyto-081211-173005.

27. Xu J, Zhang YZ, Zhang PF, Trivedi P, Riera N, Wang YY, et al. The structure and function of the global citrus rhizosphere microbiome. Nat Commun. 2018:9(1):4894. https://doi.org/10.1038/s41467-018-07343-2.

28. Bulgarelli D, Garrido-Oter R, Münch PC, Weiman A, Dröge J, Pan Y, et al. Structure and function of the bacterial root microbiota in wild and domesticated barley. Cell Host Microbe. 2015;17(3):392-403. https://doi. org/10.1016/j.chom.2015.01.011.

29. Sunagawa S, Coelho LP, Chaffron S, Kultima JR, Labadie K, Salazar G, et al. Structure and function of the global ocean microbiome. Science. 2015;348(6237):1261359. https://doi.org/10.1126/science.1261359.

30. Morgan XC, Huttenhower C. Chapter 12: human microbiome analysis. PLoS Comput Biol. 2012;8(12):e1002808. https://doi.org/10.1371/journ al.pcbi. 1002808.

31. Walsh AM, Crispie F, O'Sullivan O, Finnegan L, Claesson MJ, Cotter PD. Species classifier choice is a key consideration when analysing lowcomplexity food microbiome data. Microbiome. 2018;6(1):50. https://doi. org/10.1186/s40168-018-0437-0.

32. Zhang $Y Z, X u$ J, Riera N, Jin T, Li JY, Wang N. Huanglongbing impairs the rhizosphere-to-rhizoplane enrichment process of the citrus root-associated microbiome. Microbiome. 2017;5(1):97. https://doi.org/10.1186/ s40168-017-0304-4.

33. Heuberger H, Bauer R, Friedl F, Heubl G, Hummelsberger J, Nögel R, et al. Cultivation and breeding of Chinese medicinal plants in Germany. Planta Med. 2010;76(17):1956-62. https://doi.org/10.1055/s-0030-1250528.

34. Zhang BG, Peng Y, Zhang Z, Liu HT, Qi YD, Liu S, et al. GAP production of TCM herbs in China. Planta Med. 2010;76(17):1948-55. https://doi. org/10.1055/s-0030-1250527.

35. Dong LL, Li Y, Xu J, Yang J, Wei GF, Shen L, et al. Biofertilizers regulate the soil microbial community and enhance Panax ginseng yields. Chin Med. 2019;14:20. https://doi.org/10.1186/s13020-019-0241-1.

36. Dong LL, Xu J, Li Y, Fang HL, Niu WH, Li XW, et al. Manipulation of microbial community in the rhizosphere alleviates the replanting issues in Panax ginseng. Soil Biol Biochem. 2018;125:64-74. https://doi. org/10.1016/j.soilbio.2018.06.028.

37. Langmead B, Salzberg SL. Fast gapped-read alignment with Bowtie 2. Nat Methods. 2012;9(9):357-9. https://doi.org/10.1038/nmeth.1923.

38. Hyatt D, Chen GL, Locascio PF, Land ML, Larimer FW, Hauser LJ. Prodigal: prokaryotic gene recognition and translation initiation site identification. BMC Bioinformatics. 2010;11:119. https://doi. org/10.1186/1471-2105-11-119.

39. Fu LM, Niu BF, Zhu Z, Wu ST, Li WZ. CD-HIT: accelerated for clustering the next-generation sequencing data. Bioinformatics. 2012;28(23):3150-2. https://doi.org/10.1093/bioinformatics/bts565.

40. Buchfink B, Xie C, Huson DH. Fast and sensitive protein alignment using DIAMOND. Nat Methods. 2015;12(1):59-60. https://doi.org/10.1038/ nmeth3176.

41. Huson DH, Auch AF, Qi J, Schuster SC. MEGAN analysis of metagenomic data. Genome Res. 2007;17(3):377-86. https://doi.org/10.1101/gr.59691 07.

42. Mendes R, Kruijt M, de Bruijn I, Dekkers E, van der Voort M, Schneider $J H M$, et al. Deciphering the rhizosphere microbiome for disease-suppressive bacteria. Science. 2011;332(6033):1097-100. https://doi.org/10.1126/ science. 1203980.

43. Tao J, Wang YY, Huang YY, Xu J, Zhang PF, Wang N, et al. Taxonomic structure and functional association of foxtail millet root microbiome. Gigascience. 2017;6(10):1-12. https://doi.org/10.1093/gigascience/gix08 9.

44. Franke-Whittle IH, Manici LM, Insam H, Stres B. Rhizosphere bacteria and fungi associated with plant growth in soils of three replanted apple orchards. Plant Soil. 2015;395(1-2):317-33. https://doi.org/10.1007/s1110 4-015-2562-x.

45. Shi WC, Li MC, Wei GS, Tian RM, Li CP, Wang B, et al. The occurrence of potato common scab correlates with the community composition and function of the geocaulosphere soil microbiome. Microbiome. 2019;7(1):14. https://doi.org/10.1186/s40168-019-0629-2. 
46. Calvo P, Nelson L, Kloepper JW. Agricultural uses of plant biostimulants. Plant Soil. 2014;383(1-2):3-41. https://doi.org/10.1007/s1110 4-014-2131-8

47. Vejan P, Abdullah R, Khadiran T, Ismail S, Nasrulhaq Boyce A. Role of plant growth promoting rhizobacteria in agricultural sustainability-a review. Molecules. 2016;21(5):573. https://doi.org/10.3390/molecules21050573.

48. Kargi F, Eker S. Performance of rotating perforated tubes bioflm reactor in biological wastewater treatment. Enzyme Microb Technol. 2003;32(34):464-71. https://doi.org/10.1016/S0141-0229(02)00323-X.

49. Hashemi S, Han M, Kim T. Optimization of fertilization characteristics of urine by addition of Nitrosomonas europaea bio-seed. J Sci Food Agr. 2016;96(13):4416-22. https://doi.org/10.1002/jsfa.7652.

50. Egamberdiyeva D. The effect of plant growth promoting bacteria on growth and nutrient uptake of maize in two different soils. Appl Soil Ecol. 2007;36(2):184-9. https://doi.org/10.1016/j.apsoil.2007.02.005.

51. Upadhyay SK, Singh JS, Saxena AK, Singh DP. Impact of PGPR inoculation on growth and antioxidant status of wheat under saline conditions. Plant Biol. 2012;14(4):605-11. https://doi.org/10.1111/j.1438-8677.2011.00533.x.

52. Li XG, Ding CF, Zhang TL, Wang XX. Fungal pathogen accumulation at the expense of plant-benefcial fungi as a consequence of consecutive peanut monoculturing. Soil Biol Biochem. 2014;72:11-8. https://doi. org/10.1016/j.soilbio.2014.01.019.

53. Ren HJ, Su Y, Zhang JX, Pan HY, Chen BY, Wang Y. Recombinant protein, AlnA, combined with transgenic alfalfa remediates polychlorinated biphenyl-contaminated soils: efciency and rhizosphere microbial community response. Biotechnol Lett. 2016;38(11):1893-901. https://doi. org/10.1007/s10529-016-2169-1.

54. She SY, Niu JJ, Zhang C, Xiao YH, Chen W, Dai LJ, et al. Signifcant relationship between soil bacterial community structure and incidence of bacterial wilt disease under continuous cropping system. Arch Microbiol. 2017;199(2):267-75. https://doi.org/10.1007/s00203-016-1301-x.

55. Galán JE, Collmer A. Type III secretion machines: bacterial devices for protein delivery into host cells. Science. 1999;284(5418):1322-8. https:// doi.org/10.1126/science.284.5418.1322.

56. Eijsink VGH, Axelsson L, Diep DB, Håvarstein LS, Holo H, Nes IF. Production of class II bacteriocins by lactic acid bacteria; an example of biological warfare and communication. Antonie Van Leeuwenhoek. 2002:81(14):639-54. https://doi.org/10.1023/A:1020582211262.
57. Tseng TT, Tyler BM, Setubal JC. Protein secretion systems in bacterial-host associations, and their description in the Gene Ontology. BMC Microbiol. 2009;9(Suppl 1):S2. https://doi.org/10.1186/1471-2180-9-S1-S2.

58. Loria R, Bukhalid RA, Fry BA, King RR. Plant pathogenicity in the genus Streptomyces. Plant Dis. 1997;8:836-46. https://doi.org/10.1094/ PDIS.1997.81.8.836.

59. King RR, Lawrence $\mathrm{CH}$, Calhoun LA. Chemistry of phytotoxins associated with Streptomyces scabies the causal organism of potato common scab. J Agric Food Chem. 1992;40(5):834-7. https://doi.org/10.1021/jf00017a025.

60. Healy FG, Krasnoff SB, Wach M, Gibson DM, Loria R. Involvement of a cytochrome P450 monooxygenase in thaxtomin a biosynthesis by Streptomyces acidiscabies. J Bacteriol. 2002;184(7):2019-29. https://doi. org/10.1128/jb.184.7.2019-2029.2002.

61. Kers JA, Wach MJ, Krasnoff SB, Widom J, Cameron KD, Bukhalid RA, et al. Nitration of a peptide phytotoxin by bacterial nitric oxide synthase. Nature. 2004;429(6987):79-82. https://doi.org/10.1038/nature02504.

62. Barry SM, Kers JA, Johnson EG, Song LJ, Aston PR, Patel B, et al. Cytochrome P450-catalyzed L-tryptophan nitration in thaxtomin phytotoxin biosynthesis. Nat Chem Biol. 2012;8(10):814-6. https://doi. org/10.1038/nchembio.1048.

63. Posas F, Takekawa M, Saito H. Signal transduction by MAP kinase cascades in budding yeast. Curr Opin Microbiol. 1998;1(2):175-82. https://doi. org/10.1016/s1369-5274(98)80008-8.

64. Zhao XH, Mehrabi R, Xu JR. Mitogen-activated protein kinase pathways and fungal pathogenesis. Eukaryot Cell. 2007;6(10):1701-14. https://doi org/10.1128/EC.00216-07.

65. Negri I. Wolbachia as an "infectious" extrinsic factor manipulating host signaling pathways. Front Endocrinol. 2012;2:115. https://doi. org/10.3389/fendo.2011.00115.

66. Regan JC, Brandão AS, Leitão AB, Dias ARM, Sucena E, Jacinto A, et al. Steroid hormone signaling is essential to regulate innate immune cells and fight bacterial infection in Drosophila. PLoS Pathog. 2013;9(10):e1003720. https://doi.org/10.1371/journal.ppat.1003720.

\section{Publisher's Note}

Springer Nature remains neutral with regard to jurisdictional claims in published maps and institutional affiliations.
Ready to submit your research? Choose BMC and benefit from:

- fast, convenient online submission

- thorough peer review by experienced researchers in your field

- rapid publication on acceptance

- support for research data, including large and complex data types

- gold Open Access which fosters wider collaboration and increased citations

- maximum visibility for your research: over $100 \mathrm{M}$ website views per year

At BMC, research is always in progress.

Learn more biomedcentral.com/submissions 